\title{
Modeling Non-Equilibrium Dynamics and Saturable Absorption Induced by Free Electron Laser Radiation
}

\author{
Keisuke Hatada *,t and Andrea Di Cicco \\ Physics Division, School of Science and Technology, University of Camerino, I-62032 Camerino (MC), Italy; \\ andrea.dicicco@unicam.it \\ * Correspondence: keisuke.hatada.gm@gmail.com \\ † Current address: Department Chemie, Ludwig-Maximilians-Universität München, 81377 München, Germany
}

Received: 31 May 2017; Accepted: 27 July 2017; Published: 9 August 2017

\begin{abstract}
Currently available X-ray and extreme ultraviolet free electron laser (FEL) sources provide intense ultrashort photon pulses. Those sources open new exciting perspectives for experimental studies of ultrafast non-equilibrium processes at the nanoscale in condensed matter. Theoretical approaches and computer simulations are being developed to understand the complicated dynamical processes associated with the interaction of FEL pulses with matter. In this work, we present the results of the application of a simplified three-channel model to the non-equilibrium dynamics of ultrathin aluminum films excited by FEL radiation at 33.3, 37 and $92 \mathrm{eV}$ photon energy. The model includes semi-classical rate equations coupled with the equation of propagation of the photon wave packets. X-ray transmission measurements are found to be in agreement with present simulations, which are also able to shed light on temporal dynamics (in the fs range) in nano-sized Al films strongly interacting with the photon pulse. We also expanded our non-linear model, explicitly including the two-photon absorption cross-section and the effect of including electron heating for reproducing transmission measurements.
\end{abstract}

Keywords: X-ray free electron laser; saturation phenomena; nonlinear optics

\section{Introduction}

Over the past decade, X-ray and extreme ultraviolet free electron laser (FEL) sources have been developed, providing a source of extremely brilliant and ultrafast photon pulses. The present facilities include FELs in the extreme ultra-violet (EUV) and soft X-ray ranges such as FLASH (Hamburg) [1] and FERMI@Elettra (Trieste) [2], and in the hard X-ray range such asLCLS (Stanford) [3], SACLA (Spring-8) [4], and the European XFEL (presently under construction, Hamburg).

Typically, FEL photon pulses show durations in the 10-100 fs range, contain a large number of photons $\left(10^{10}-10^{15}\right)$ with a limited wavelength band width, depending on the pulse generation mechanism. Using suitable optics, the pulse spot dimensions can be reduced to $10-100 \mathrm{~mm}^{2}$ or less. In such extreme conditions, pulse fluences can exceed $100 \mathrm{~J} / \mathrm{cm}^{2}$, leading to the observation of non-linear optical processes in condensed matter such as saturation phenomena, two photon absorption, ultrafast electron, and lattice heating (see [5] and the references therein).

Non-linear effects are quite familiar in optical laser science, while in the EUV and X-ray energy regime, investigations are still in development both experimentally and theoretically. There are several differences among the optical laser and EUV /X-ray radiation interaction with matter, including the penetration depth, the energy deposited, and the lifetime of the excited states. An important feature is exactly the latter, because for X-ray excitations, the lifetime of the excited state is in the femtosecond range (core-hole lifetime). Intense and ultrashort FEL pulses allow us to perform experiments for which the pulse width is of the same order of the core-hole lifetime at fluences for which non-linear effects are not negligible. 
Different techniques are used at FEL facilities, including transmission and scattering experiments and pump-and-probe studies using two ultrashort pulses (optical or X-ray). In many cases, simplified approaches are used for modeling the interaction process, basically neglecting the finite time widths and spatial dimensions of the pulses. Although efficient collisional-radiative codes describing dense plasma states are currently available (SCFLY, see [6] and references therein), the complicated dynamical processes associated with the interaction of matter with photon pulses are usually not taken into explicit account. For FEL ultrashort pulses and nanoscale materials, the finite dimensions are expected to play a role, and a detailed modeling of the dynamical pulse-matter interaction appears to be necessary. In particular, reliable theoretical models are needed both for a solid interpretation of the experimental data (transmission, scattering, and so on) and for modeling the evolution of the sample status during the excitation process (transient conditions, local temperature).

In principle, transmission measurements are probably the easiest and cleanest experiments that can be performed using FEL radiation, but they also imply several important difficulties to be overcome both for their practical realization and interpretation of results. The importance of developing proper models can be appreciated by looking first at the results of Nagler et al. [7], in which saturable soft X-ray absorption $(92 \mathrm{eV})$ of an ultrathin aluminum foil was obtained. Those results were followed by other experiments at lower (23.7-37 eV) [8] and higher photon energy (1540-1870 eV) [9], both indicating the importance of accounting for electron heating phenomena at high fluence. Further experiments in the hard X-ray range $(7.1 \mathrm{keV})$ confirmed the existence of important non-linear phenomena with increasing transmission of a factor of 10 and substantial shifts of the absorption edge in solid iron [10].

Those results indicate that the description of photon-matter interaction at high intensities requires specific models to be devised, accounting for the various effects contributing to a modification of the X-ray and EUV absorption cross-section (relaxation of final state, ultrafast electron heating, and so on). There have been several theoretical and computational works on Al EUV and X-ray FEL intense pulse absorption. Examples range from "dynamical" (small cluster) [11,12] to "steady" model [13,14] approaches, including application of the SCFLY code [15].

In previous works, we have developed a simple three-channel model (involving ground, excited and relaxed states) for calculating the transmission of ultrathin metal films for increasing photon fluences using FEL radiation up to the saturation limit [16]. The model was conceived to provide a reliable and physically-intuitive calculation scheme for saturable absorption, also allowing direct inspection of the interaction dynamics including size effects of the pulse width and finite thickness of the absorbing medium at the nanometric scale. The three-channel model was found to provide simulations in excellent agreement with EUV transmission data in an extended range of pulse fluence $\left(0-200 \mathrm{~J} / \mathrm{cm}^{2}\right)[8,16]$. Simulations allow us direct estimates of time and space profiles for the photon absorption, and a benchmark for calculating observables of interest (transmission) and the deposited energy in nanosized condensed matter for evaluation, for example, of local temperatures. The effects of a local increase of the electron temperature can also be introduced in the calculations as reported in Reference [8]. In this work, we extend previous results for the calculation of EUV transmission of ultrashort pulses through Al films of nanometric thickness. We show the details of the temporal dynamics of the photon-matter interaction at fs resolution, also including possible multi-photon absorption and electron-heating effects.

\section{Model for Ultrafast Transmission Measurements}

\subsection{Three-Channel Model}

In our recent work [16], we developed a phenomenological three-state model which is able to reproduce saturation phenomena related to the increased transmission in the high fluence regime. We refer to the original publication [16] for some more details on the computational model. In brief, the model describes the variation of the density of occupation numbers $N_{1}, N_{2}$ and $N_{3}$ of three 
exemplary many-body states (ground $|1\rangle$, excited $|2\rangle$ and an intermediate relaxed state $|3\rangle$ ) by a set of rate equations with proper constraints:

$$
\begin{gathered}
\frac{d N_{1}(z, t)}{d t}=\frac{g(z, t) I(z, t)}{h v}+\frac{N_{2}(z, t)}{\tau_{21}}+\frac{N_{3}(z, t)}{\tau_{31}} \\
\frac{d N_{2}(z, t)}{d t}=-\frac{g(z, t) I(z, t)}{h v}-\frac{N_{2}(z, t)}{\tau_{21}}-\frac{N_{2}(z, t)}{\tau_{23}} \\
\frac{d N_{3}(z, t)}{d t}=\frac{1}{\tau_{23}} N_{2}(z, t)-\frac{1}{\tau_{31}} N_{3}(z, t) \\
g(z, t)=\sigma(T)\left(N_{2}(z, t)-N_{1}(z, t)\right) \\
N=N_{1}(z, t)+N_{2}(z, t)+N_{3}(z, t)
\end{gathered}
$$

In this set of equations, the occupation numbers depend thus on the photon field intensity $I(z, t)$ at time $t$ and position $z$ (along the direction of propagation of the pulse), the photon absorption cross-section $\sigma$ at given photon energy $h v$, and on the relaxation times $\tau$ between the various states. In this formalism, $g(z, t)$ is an effective time- and space-dependent absorption coefficient-possibly temperature-dependent-that can be considered constant for linear absorption (Lambert-Beer law) at a given temperature $T$. From the condition of conservation of the total number of the states Equation (5), we just need to solve couples of rate equations.

The set of rate equations are coupled with the transport condition of the incoming pulse, within the classical electrodynamics limit:

$$
\frac{d I(z, t)}{d z}+\frac{1}{c} \frac{d I(z, t)}{d t}=g(z, t) I(z, t)
$$

Within the model described by the equations above, absorption and stimulated emission by laser radiation involve transitions between ground $|1\rangle$ and excited $|2\rangle$ states. The relaxed state $|3\rangle$ represents the ensemble of all possible relaxed states reached by decay of state $|2\rangle$. It can decay to state $|1\rangle$ by emitting a photon or through other processes.

\subsection{Computational Details}

We describe here the way of solving the set of coupled equations numerically. For the discretization of Equation (6), we employed the forward differentiation, as the equations can be solved numerically following the dynamics of the pulse, using discretized grids with $\Delta t=0.4$ as and $\Delta z=1.2 \mathrm{~nm}$, which satisfy the Courant condition,

$$
c \leq \frac{\Delta z}{\Delta t}
$$

so that the Upward differencing' method [17] can be applied safely to Equation (6):

$$
\frac{I\left(z_{j}, t_{n+1}\right)-I\left(z_{j}, t_{n}\right)}{c \Delta t}=-\frac{I\left(z_{j}, t_{n}\right)-I\left(z_{j-1}, t_{n}\right)}{\Delta z}+g\left(z_{j}, t_{n}\right) I\left(z_{j}, t_{n}\right)
$$

In Equation (8), $j$ runs along the incident direction of the FEL pulse inside the sample and $n$ represents the discrete time grid.

In the rate Equation (5), we just used the Euler method; thus, for example, we obtain the following expression for $\mathrm{N}_{2}$ : 


$$
\frac{N_{2}\left(z_{j}, t_{n+1}\right)-N_{2}\left(z_{j}, t_{n}\right)}{\Delta t}=-\frac{g\left(z_{j}, t_{n}\right) I\left(z_{j}, t_{n}\right)}{h v}-\left[\frac{1}{\tau_{21}}+\frac{1}{\tau_{23}}\right] N_{2}\left(z_{j}, t_{n}\right) .
$$

We remark that for actual calculations concerning intense ultrafast pulses and nano-sized samples we need extremely small $\Delta t=0.4$ and $\Delta z=1.2 \mathrm{~nm}$, so that efficient algorithms like those mentioned are needed for best accuracy and lower computing times.

For the initial pulse intensity $I_{0}$, we assumed a Gaussian time distribution:

$$
I_{0}(z, t)=I_{0}^{\max } e^{-\frac{\left(z-c\left(t+\mu_{0}\right)\right)^{2}}{2\left(c \sigma_{t}\right)^{2}}}
$$

where $\mu_{0}$ and $\sigma_{t}$ are the mean and standard deviation of the photon pulse time distribution. $I_{0}^{\max }$ corresponds to the maximum intensity of initial beam. For a given pulse shape, $\sigma_{t}$ is estimated looking from the full-width at half maximum FWHM $=2 \sqrt{2 \ln 2} \sigma_{t}$. The window of the pulse in time is set at just double of FWHM (namely $30 \mathrm{fs}$ ), multiplied by the speed of light. The origin of $z$ is set at the surface of a sample. The three-channel model is implemented in a Fortran 90 code using simple instructions and running on any current operating systems and with any Fortran compiler.

\section{Results and Discussion}

\subsection{Transmission by Ultrashort Pulse of Photons with $92 \mathrm{eV}$ for Al Foil}

As mentioned above, saturable absorption using FEL pulses was first observed in a pioneering experiment by Nagler et al. [7], in which single-shot transmission data of a $53 \mathrm{~nm}$ Al foil were collected using $92 \mathrm{eV}$ ultra-short (15 fs) photon pulses up to fluences in the $200 \mathrm{~J} / \mathrm{cm}^{2}$ range. FEL pulses are thus able to excite $\mathrm{Al} 2 \mathrm{p}$ core electrons (binding energies are 72.7 and $73.1 \mathrm{eV}$ ), and the lifetime of the excited core-hole state is $40 \mathrm{fs}$ [18].

Several approximations can be used for calculating X-ray transmission using the model reported in the previous section. In particular, fast calculations can be performed considering two opposite limits, namely: (i) a long pulse limit in which the pulse width is much longer than the lifetime of the excited state; (ii) a short pulse limit in which the lifetime is much longer than the pulse width.

In both limits, the system under the photon field is assumed to be in a steady-state. Hence, the time dependency is neglected; that is to say, the time derivative of the occupation numbers and the intensity (see Equation (5)) become zero. The details of the models are described in Appendixes A and B.

We have performed calculations of $\mathrm{Al}$ transmission comparing the results of the long and short pulse limits with those of the three-channel model including the dynamics of the pulse-matter interaction. In Figure 1, we show the comparison between the different models and experimental data [7]. For the dynamical three-channel and long pulse models, we included the ground state $|1\rangle$, excited state $|2\rangle$ and relaxed state $|3\rangle$. We assumed that the lifetime of the relaxed state $\tau_{31}$ is the same as the one of the excited state $\tau_{21}$ (namely, $40 \mathrm{fs}$ ), while the relaxation time to the relaxed state $\tau_{32}$ is $1 \mathrm{fs}$, corresponding to the electron mean free path [7]. We also tested a short pulse model, which represents just the opposite limit of the long pulse model. This model assumes that the pulse width is much shorter than the lifetime of the excited state, so that decay of excited state is neglected. Without decay process, the state cannot access the relaxed state, thus the model reduces to a two channel model.

As shown in Figure 1, all four models show an increase in transmission with the fluence until reaching the saturation at extremely high fluence above $200 \mathrm{~J} / \mathrm{cm}^{2}$. In the low-fluence limit, the calculations show the convergence to the linear transmission (Lambert-Beer law). The four models result in a completely different transmission at intermediate fluences, and the long pulse with and without the relaxed state and short pulse limits fail to reproduce the experimental data. These static models imply remarkably increased transmission in the $2-100 \mathrm{~J} / \mathrm{cm}^{2}$, anticipating the onset of saturable absorption of at least one order of magnitude. On the other hand, the dynamical three-channel 
model-which explicitly considers the time evolution of the occupation numbers along the pulse direction - is found to be in very good agreement with the experimental data.

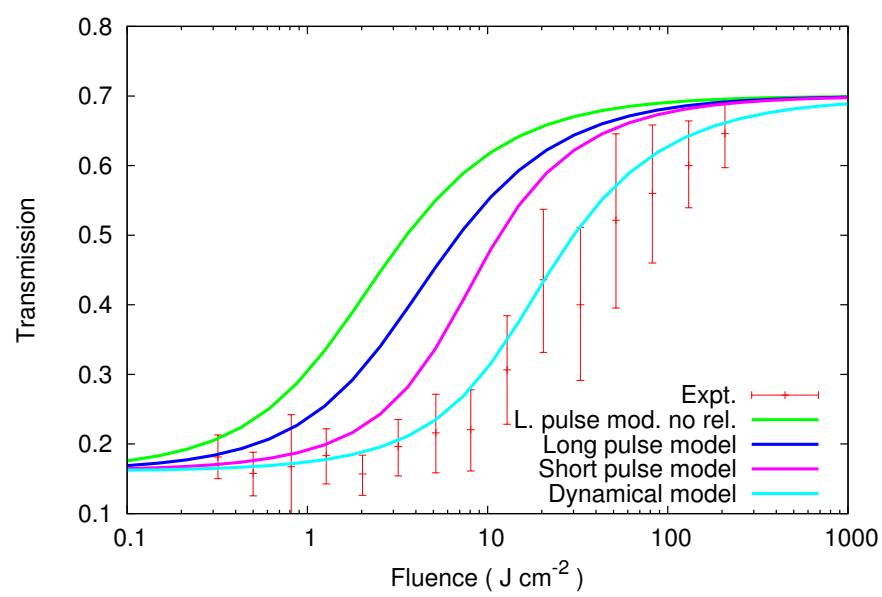

Figure 1. XFEL pulse transmission for $\mathrm{Al}$ thin film $(53 \mathrm{~nm})$ at $92 \mathrm{eV}$ photon energy. The green and blue solid lines indicate long pulse model without (no rel.) and with an intermediate relaxed state; that is, the model of the green line is just a two-channel model. The pink solid line is for the short pulse model. The light blue solid line corresponds to the three-channel dynamical model. The dynamical model agrees very well with the experimental (Expt.) data [7].

As will be shown in the next Section, the steady condition of the long pulse model is not achieved at the beginning and end of the incident pulse, while a "steady" condition can be obtained only above a threshold intensity of the pulse taking account of its shape.

\subsection{Non-Equilibrium Dynamics in Al Thin Film}

In the previous section, we have shown that the three-channel model describes the saturation phenomena of X-rays of ultra short pulse well. In this part, we show the detail of the dynamics in the Al thin film during $60 \mathrm{fs}$. Figure 2 shows the results of time dependence of the density of occupation numbers normalized by the total number $N$ at the end face of $\mathrm{Al}$ thin $(53 \mathrm{~nm})$ film. The fluences $F$ are $1.5,15$ and $150 \mathrm{~J} / \mathrm{cm}^{2}$ for (a), (b) and (c), respectively. In the calculation, we estimated that the additional absorption by both sides of oxidized Al layers [7] reduces the pulse intensity by $70 \%$. In the figures, the results obtained from the dynamical three-channel model is shown with solid lines, and static long pulse model with the dotted line. In Figure $2 \mathrm{a}$, we see that the results of the dynamical model tend asymptotically to reach the static limit. However the time of irradiation is not sufficient to reach those occupation number limits, which is around $10 \%$ of the population for the excited state. The dynamical model shows that the system is still highly populated by the ground state. In fact, this can be confirmed looking at Figure 1, where we see that the transmission of long pulse model already shows a difference from the linear optical transmission, while the experimental and the dynamical ones are still well below the saturation threshold. For higher fluence at $15 \mathrm{~J} / \mathrm{cm}^{2}$, in Figure 2b, we see that the ratio of the occupation number of the excited state is much increased, such that the linear model (Lambert-Beer law) which works for the ground state fails. This failure is confirmed experimentally by the observation of growth of the transmission in Figure 1. In Figure 2c, we see that by increasing the pulse intensity the occupation of the ground state is strongly decreased, while most electrons are in the excited state. Under these conditions, the increment of the transparency and saturable absorption as the consequence of this are realized for this $\mathrm{Al}$ film. In this fluence range, a population inversion corresponding formally to negative temperatures (in equilibrium statistical mechanics terms) is realized. This phenomenon is associated with the existence of the relaxed state $|3\rangle$, which does not participate in the photon absorption and simulated emission. In our calculations, 
the system returns to the ground state exponentially at the end of the short photon pulse (times larger than 60 fs in Figure 2).

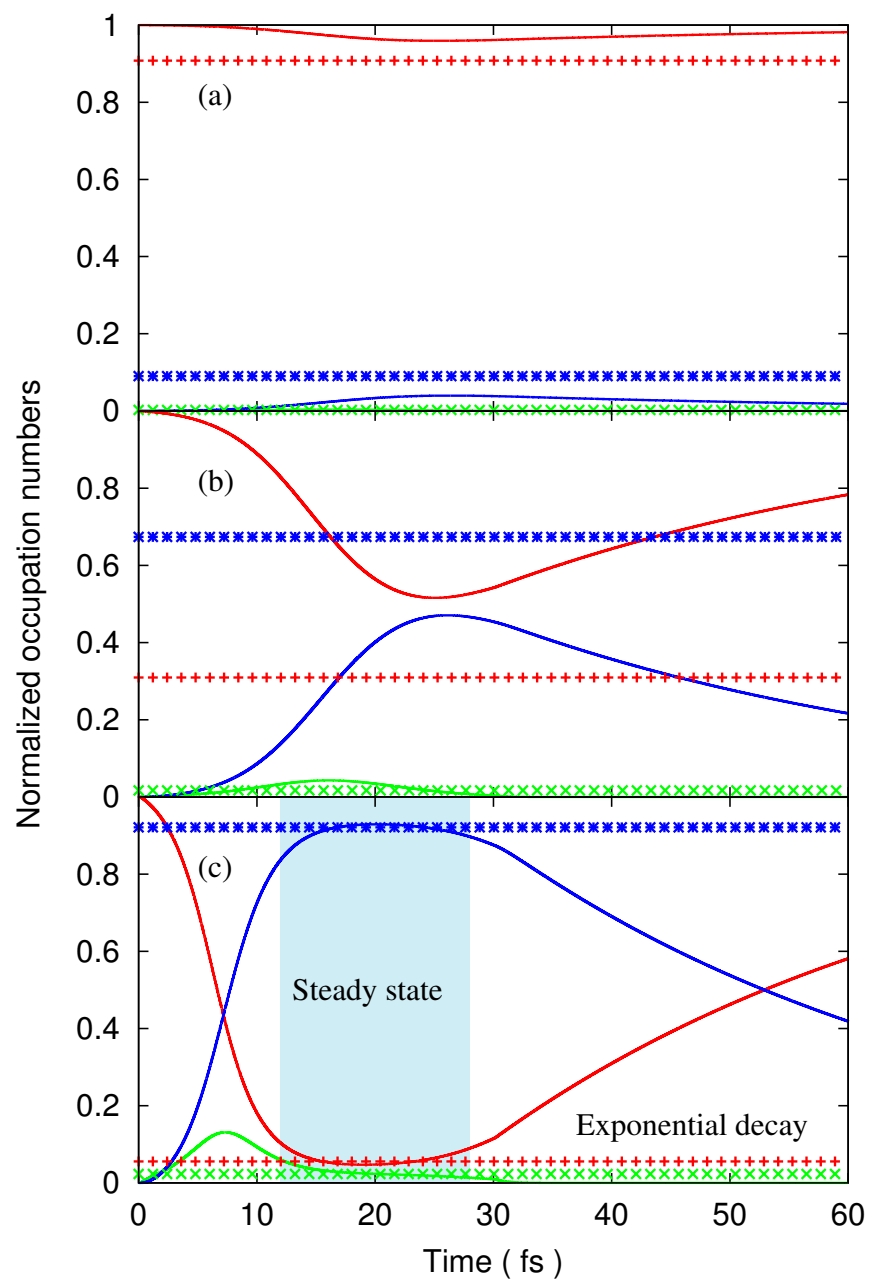

Figure 2. Time dependence of the density of occupation numbers (normalized by the total number $N$ ) at the end face of $\mathrm{Al}$ thin film. The conditions are the same as in Figure 1. The fluences of incoming Gaussian pulses are 1.5, 15 and $150 \mathrm{~J} / \mathrm{cm}^{2}$ from the top to the bottom of three figures, respectively. The solid curves and dotted lines are estimated by the three-channel model and long pulse model, respectively. The red color indicates the ground state $|1\rangle$, the blue one the excited state $|2\rangle$ and the green one the relaxed state $|3\rangle$. After some time, the curves asymptotically approach the steady state of the long pulse model. In the region of the light blue shade, the system has reached a steady state. After about $30 \mathrm{fs}$ (the total pulse width), the excited state decays exponentially. The averaged experimental data are indicated by red points with the errors.

\subsection{Simulations for Two Photon Absorption}

Another source of non-linear optical phenomena in FEL absorption studies is represented by the presence of two photon absorption (TPA) effects. The presence of TPA can be obtained at extremely large fluences, but the order of two photon absorption cross-section is predicted to be rather small, with typical values in the $10^{-50} \mathrm{~cm}^{4} \mathrm{~s}$ range. In order to estimate the size of this effect quantitatively, we apply the following set of equations:

$$
\frac{d N_{1}(z, t)}{d t}=\frac{g(z, t) I(z, t)}{h v}+\frac{g_{2}(z, t) I^{2}(z, t)}{h v}+\frac{N_{2}(z, t)}{\tau_{21}}+\frac{N_{T}(z, t)}{\tau_{T 1}}
$$




$$
\begin{gathered}
\frac{d N_{2}(z, t)}{d t}=-\frac{g(z, t) I(z, t)}{h v}-\frac{N_{2}(z, t)}{\tau_{21}} \\
\frac{d N_{T}(z, t)}{d t}=-\frac{g_{2}(z, t) I^{2}(z, t)}{h v}-\frac{1}{\tau_{T 1}} N_{T}(z, t) \\
\frac{d I(z, t)}{d z}+\frac{1}{c} \frac{d I(z, t)}{d t}=g(z, t) I(z, t)+g_{2}(z, t) I^{2}(z, t) \\
g(z, t)=\sigma\left(N_{2}(z, t)-N_{1}(z, t)\right) \\
g_{2}(z, t)=\frac{\sigma_{2}}{h v}\left(N_{T}(z, t)-N_{1}(z, t)\right) \\
N=N_{1}(z, t)+N_{2}(z, t)+N_{T}(z, t)
\end{gathered}
$$

where $\sigma_{2}$ is the two photon absorption cross-section (in $10^{-50} \mathrm{~cm}^{4} \mathrm{~s}$ ). The new term $g_{2}(z, t) I^{2}(z, t)$ in Equation (14) contributes to the increase (or decrease) of the intensity through the two photon absorption and stimulated emission. We introduced a new state $|T\rangle$ which is accessible by TPA. This state is only connected to the ground state $|1\rangle$ by two photon absorption and stimulated emission. The lifetime of state $|T\rangle$ to the ground state $|1\rangle, \tau_{T 1}$ is set to be the same as $\tau_{21}$, which is $40 \mathrm{fs}$. In order to see the effects of TPA only, we did not introduce the relaxed state. The ionization energy of the ionic state with a hole in $2 p$ orbital is $93 \mathrm{eV}$ [7], which is higher than the photon energy, $92 \mathrm{eV}$, so we do not need to include the sequential two photon absorption.

We report calculations with four different values of $\sigma_{2}$; namely $0,10^{-50}, 10^{-49}$ and $10^{-48} \mathrm{~cm}^{4} \mathrm{~s}$. $\sigma_{2}=0$ reduces to a single photon absorption two-channel model. For a limit of infinitely long pulse, it corresponds to the long pulse model without relaxed state (green line) in Figure 1. $\sigma_{2}=10^{-49}$ and $10^{-48} \mathrm{~cm}^{4}$ s represent large cross-sections for TPA and are probably not realistic, but we show the results to check the tendency for TPA effects in X-ray transmission.

In Figure 3, we show the transmission of X-ray pulse for Al thin film with the same condition as in Figure 1. Application of the present TPA models does not seem to reproduce the experimental results. As we see also from the ground state asymptotic model (steady-state TPA, Appendix C, Equation (A19)), TPA plays an important role at high photon intensity, decreasing the total transmission. In the high fluence regime, TPA and saturable absorption are both factors affecting the transmission.

Figure $4 \mathrm{a}-\mathrm{c}$ show the details of dynamics for the states at the end face of the $\mathrm{Al}$ thin film with $\sigma_{2}=10^{-50} \mathrm{~cm}^{4}$ s for fluences of $1.5,15$ and $150 \mathrm{~J} / \mathrm{cm}^{2}$, respectively. The conditions of the calculations are the same as in Figure 2a-c. For 1 and $10 \mathrm{~J} / \mathrm{cm}^{2}$ in Figure $4 \mathrm{a}, \mathrm{b}$, respectively, the results are similar to the previous saturation model (ratio $N_{1}: N_{2}$ ). For high fluence in Figure $4 \mathrm{c}$, the ratio of the occupation numbers of the states approaches $N_{1}: N_{2}: N_{3}=1 / 3: 1 / 3: 1 / 3$ in steady conditions (which corresponds to the highest entropy in the sense of the equilibrium statistical mechanics); therefore, the population inversion is not obtained. 


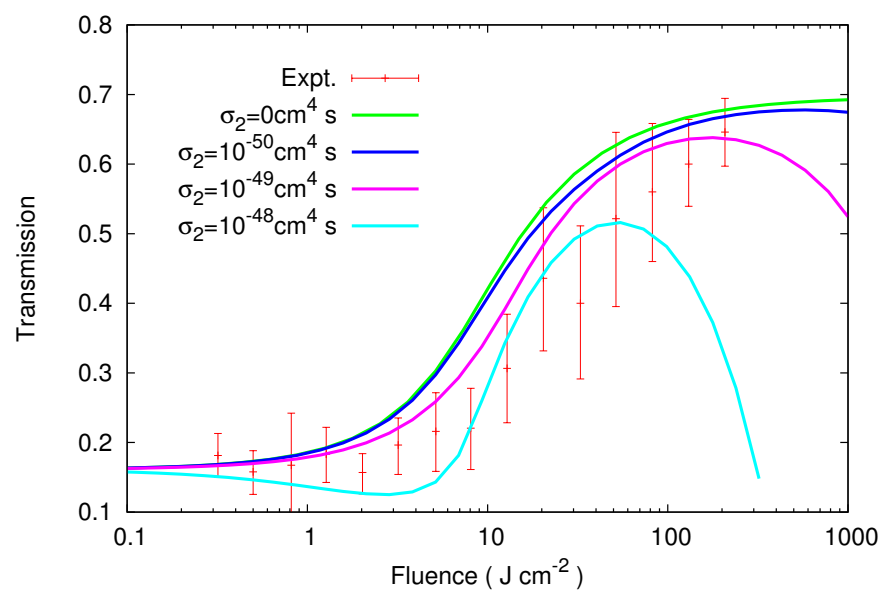

Figure 3. Transmission for Al thin film $(53 \mathrm{~nm})$ with two photon absorption (TPA) model at $92 \mathrm{eV}$ photon energy of X-ray pulse, compared with experimental data (Expt.). Four different TPA cross sections $-0,10^{-50}, 10^{-49}$ and $10^{-48} \mathrm{~cm}^{4} \mathrm{~s}$-are used to demonstrate a non-linear optical effect.

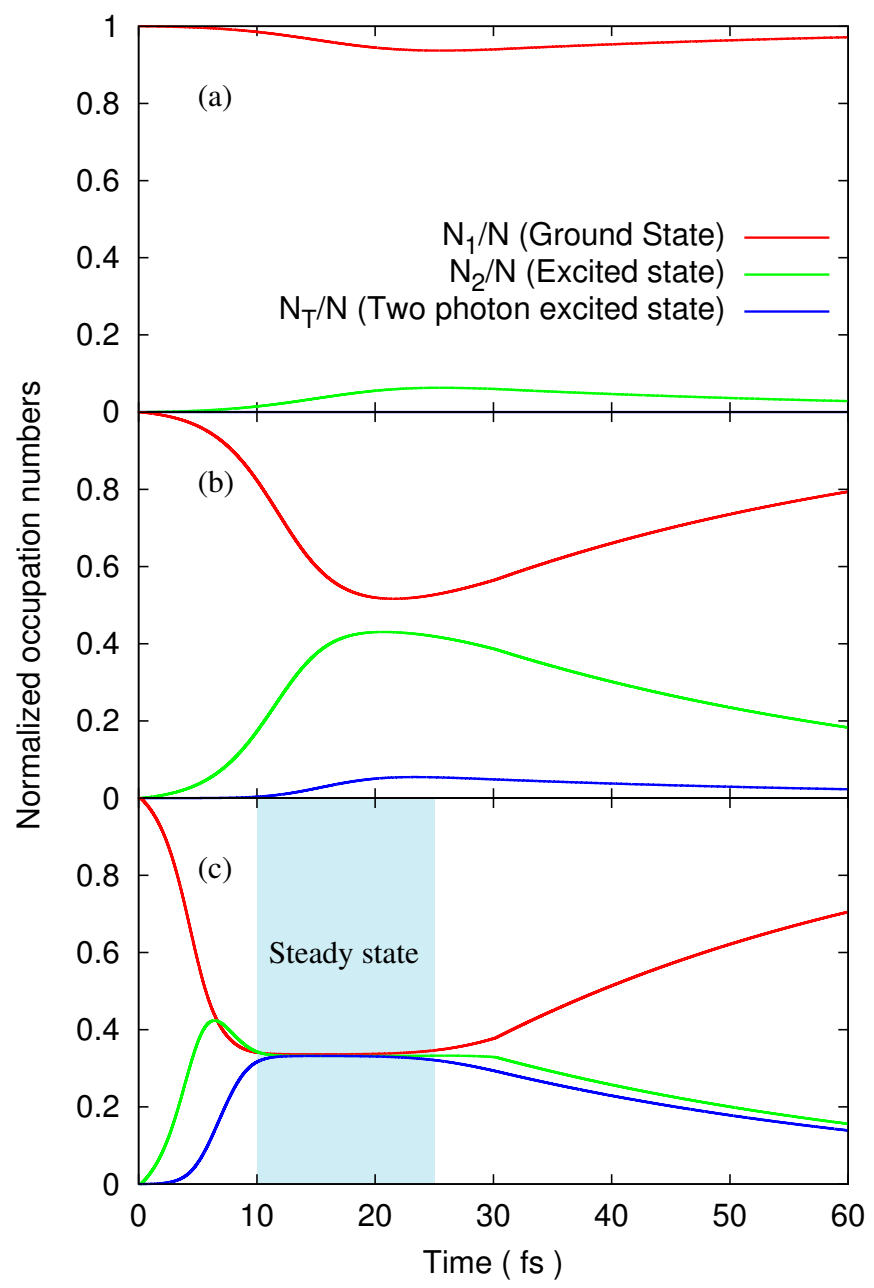

Figure 4. Time dependence of the density of occupation numbers (normalized by the total number $\mathrm{N}$ ) at the end surface of the $\mathrm{Al}$ thin film $(53 \mathrm{~nm})$, using the two photon absorption (TPA) contribution at $92 \mathrm{eV}$ photon energy (FEL pulse). The fluences of the incident pulse are $1.5,15$ and $150 \mathrm{~J} / \mathrm{cm}^{2}$ from the top to the bottom, respectively. The TPA cross-section $\sigma_{2}$ is set to $10^{-50} \mathrm{~cm}^{4} \mathrm{~s}$. 


\subsection{Transmission by Ultrafast Pulse of Photons with 33.3 and $37 \mathrm{eV}$ for Al Foil}

Another EUV transmission experiment [8] was performed at the TIMEX end station $[19,20]$ of the Elastic and Inelastic Scattering (EIS) beamline [21], using the FERMI@Elettra FEL-1 source, a seeded FEL providing clean, tunable [2], and intense subpicosecond (100 fs FWHM) photon pulses in the 19-62 eV photon energy range.

Accurate transmission measurements were carried out using unsupported self-standing 100-nm-thick $\mathrm{Al}$ foils. The maximum energy per pulse delivered by the FEL-1 source were $180 \mu \mathrm{J}$ and $130 \mu \mathrm{J}$ range at 33.3 and $37 \mathrm{eV}$ photon energy, respectively. The actual incoming fluence $F$ at sample position was estimated by measuring the area of the focal spot $\left(\sigma^{2} \approx 100 \mu \mathrm{m}^{2}\right)$ and accounting for mirror reflectivity and beamline transmission. Data were collected over several decades of fluence $F$ $\left(0.01-20 \mathrm{~J} / \mathrm{cm}^{2}\right)$ through the combined use of filters and a gas attenuator. Appreciable damage of the sample was observed after irradiation with a single FEL pulse with $F>0.1 \mathrm{~J} / \mathrm{cm}^{2}$, and single-shot transmittance measurements were carried out in the high fluence regime.

EUV transmission data were measured with unprecedented accuracy, and evidence for a nonmonotonic trend as a function of incoming fluence was found. In particular, an increased transmittance at higher fluence of about $5-10 \mathrm{~J} / \mathrm{cm}^{2}$-associated with saturable absorption effects-was accompanied by an initial decrease at intermediate fluences, as shown in Figure 5.

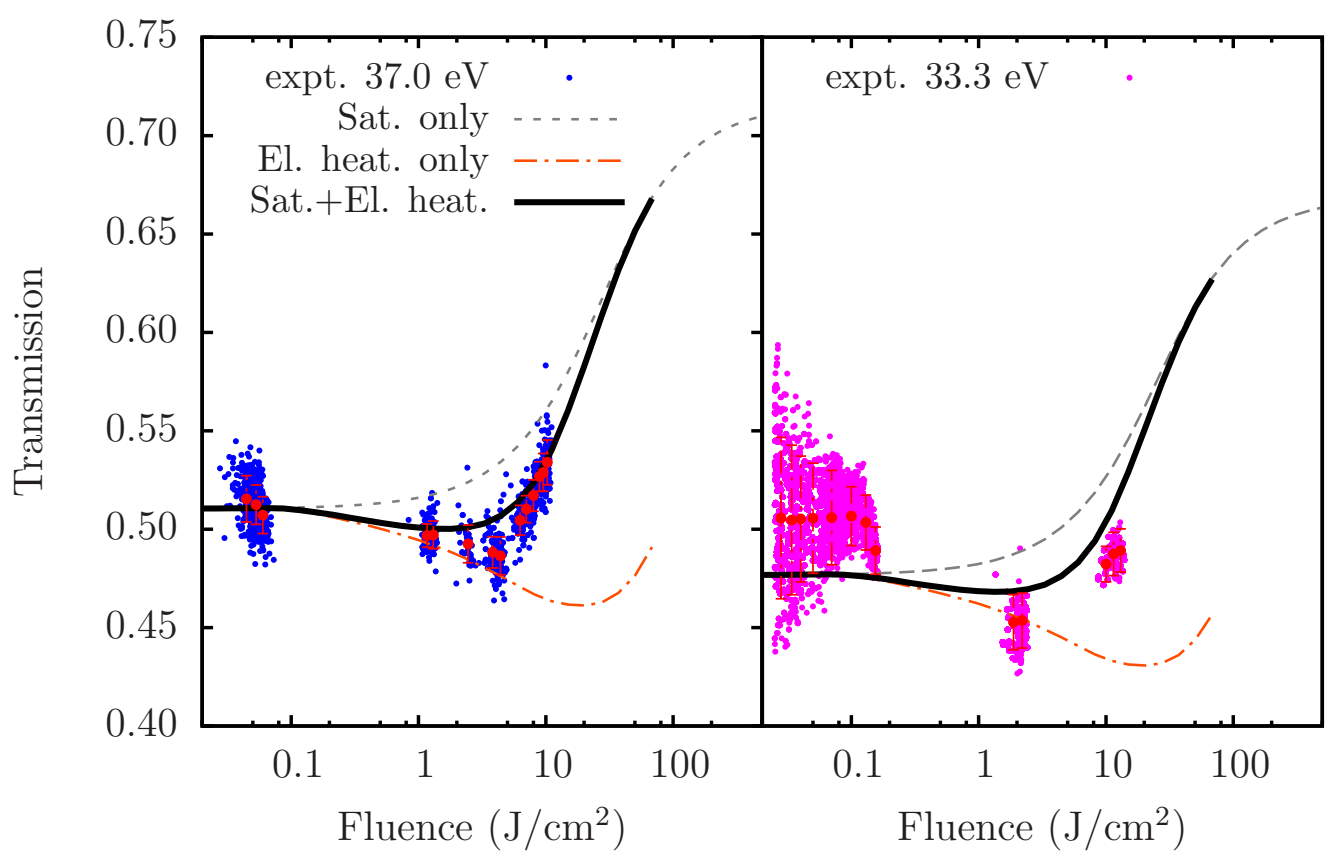

Figure 5. Experimental (expt.) EUV transmission data at $37 \mathrm{eV}$ (left panel) and $33.3 \mathrm{eV}$ (right panel) compared with different calculations, including: only optical saturation phenomena without accounting for temperature effects in the cross-section (Sat. only); only electron heating but neglecting saturation effects (El. heat. only); both electron heating and saturation effects (Sat.+El. heat.).

As we have seen in previous sections, this decrease can not be reproduced by a pure single-photon optical effect. Two-photon effects can decrease the observed transmittance under suitable conditions. However, we have found that experimental data showing a non-monotonic behaviour cannot be reproduced by realistic TPA models. The cross-section $\sigma_{2}$ for two-photon absorption should be at least on the order of $10^{-48} \mathrm{~cm}^{4} \mathrm{~s}$, two orders of magnitude larger than expected.

In a recent work done by Vinko et al. [14], detailed calculations of the cross-section for selected photon energy and electron temperatures for aluminium were reported. These calculations show clear changes in photon absorption due to the electron distribution, and in particular, a peculiar increase of absorption in an intermediate range of electron temperatures $(1-10 \mathrm{eV})$ was found. We introduced 
the results of the calculations through a temperature-dependent absorption coefficient $\sigma(T)$ with effective electron temperature $T_{e}$. For each slice of $\Delta z$ for the simulation, we estimated the local electron temperature from a Maxwell-Boltzmann model as $k_{B} \Delta T_{e}=\frac{2}{3 n_{e}} \frac{\Delta F}{\Delta z}$, where $n_{e}$ is a number of valence electron and $\Delta F$ is deposited energy per unit surface. Figure 5 shows experimental (expt.) EUV transmission data at $37 \mathrm{eV}$ (left panel) and $33.3 \mathrm{eV}$ (right panel) compared with different calculations, including: only optical saturation phenomena without accounting for temperature effects in the cross section (Sat. only); only electron heating but neglecting saturation effects (El. heat. only); both electron heating and saturation effects (Sat.+El. heat.). We see that the Sat. only model (no account for electron temperature) does not reproduce the decrease of the transmission between 1 to $5 \mathrm{~J} / \mathrm{cm}^{2}$. The El. heat. only (temperature-dependent, linear) model shows the decrease in transmittance, but the increase at high fluence is not reproduced. Finally, the Sat.+El. heat. model agrees closely with the experimental results, so for these EUV absorption experiments, electron heating is found to play an important role in understanding the initial decrease in transmittance observed in these accurate experimental data.

\section{Conclusions}

In this work we have shown the results of the application of a simplified three-channel model to the non-equilibrium dynamics of ultrathin aluminum films excited by FEL radiation at 33.3, 37 and $92 \mathrm{eV}$ photon energy. The theoretical model has been developed and implemented into a Fortran 90 code using semi-classical rate equations coupled with the equation of propagation of the photon wave packets. X-ray transmission measurements for pulses of different photon energy and fluence were found to be in agreement with present simulations. Simulations were shown to be able to shed light on temporal dynamics (in the fs range) for nano-sized Al films strongly interacting with the photon pulse. Simulations of non-equilibrium dynamics were reported for three different maximum intensities of FEL pulses corresponding to typical fluences for a system far from saturation and near the saturation threshold. We remark that the three-channel model including a relaxed state produced a population inversion state, leading to negative temperatures which is a typical consequence of non-equilibrium dynamics induced by laser. We also expanded our non-linear model, including explicitly the two-photon absorption (TPA) cross-section and the effect of including electron heating for reproducing accurate transmission measurements. Within our simplified TPA model, we have obtained the highest entropy state in the limit of high fluence, corresponding to positive temperatures (no inversion). Through the series of numerical calculations using different models reported in this article, we have shown that for the case of short EUV/X-ray pulses, compared to the lifetime of the excited state, we need to employ a dynamical (time-dependent) model. Furthermore, electron heating effects must be taken into account for a deeper level of agreement with experimental data showing saturable absorption at high fluence, as shown by a direct comparison with accurate EUV absorption data.

Acknowledgments: This work has been carried out in the framework of the TIMEX collaboration (time-resolved studies of matter under extreme and metastable conditions: http://gnxas.unicam.it/TIMEX). TIMEX was a project financed by the FERMI@Elettra FEL facility in Trieste in collaboration with the University of Camerino. Keisuke Hatada gratefully acknowledges TIMEX research grants and the authors acknowledge the COST Action MP1306 EUSpec for support.

Author Contributions: Both authors equally contributed to this article.

Conflicts of Interest: The authors declare no conflict of interest.

\section{Appendix A. Long Pulse Limit}

The set of rate equations in Equatioins (1)-(5) is simplified by using the condition of conserving the total number of the states; that is to say, substituting $N_{1}$ by $N-N_{2}-N_{3}$,

$$
\frac{d N_{2}(z, t)}{d t}=-\frac{g(z, t) I(z, t)}{h v}-\frac{N_{2}(z, t)}{\tau_{21}}-\frac{N_{2}(z, t)}{\tau_{23}}
$$




$$
\begin{gathered}
\frac{d N_{3}(z, t)}{d t}=\frac{1}{\tau_{23}} N_{2}(z, t)-\frac{1}{\tau_{31}} N_{3}(z, t) \\
g(z, t)=\sigma(T)\left[2 N_{2}(z, t)-N+N_{3}(z, t)\right]
\end{gathered}
$$

For a long pulse limit where the duration of the pulse is much longer than the lifetime of the states, the system reaches a steady state, such that the time derivatives go to zero:

$$
\frac{d N_{1}(z, t)}{d t}=\frac{d N_{2}(z, t)}{d t}=\frac{d N_{3}(z, t)}{d t}=0, \quad \frac{d I(z, t)}{d t}=0 .
$$

Thus, we can further simplify the equations

$$
\begin{gathered}
\frac{d I(z)}{d z}=\frac{g_{0}}{1+\frac{I(z)_{s}}{\alpha I_{s}}} I(z) \\
g_{0}=-\sigma(T) N \\
I_{s}=\frac{h v}{2 \sigma \tau_{21}} \\
\alpha=1+\frac{2 \tau_{21}-\tau_{31}}{2 \tau_{23}+\tau_{31}}
\end{gathered}
$$

The time-independent density of occupation numbers are:

$$
\begin{aligned}
N_{1}(z) & =N-\left(1+\frac{\tau_{31}}{\tau_{23}}\right) N_{2}(z) \\
N_{2}(z) & =\frac{1}{2} \frac{1}{1+\frac{\tau_{31}}{2 \tau_{23}}} \frac{\frac{I(z)}{\alpha I_{s}}}{1+\frac{I(z)}{\alpha I_{s}}} N \\
N_{3}(z) & =\left[1+\frac{1}{1+\frac{I(z)}{\alpha I_{s}}}\right] N .
\end{aligned}
$$

The solution of Equation (A4) is:

$$
\begin{gathered}
I(z)=I_{\text {sat }} f_{W}^{-1}\left(\frac{I_{0}}{I_{\text {sat }}} e^{\frac{I_{0}}{I_{\text {sat }}}} e^{g_{0} z}\right) \\
I_{\text {sat }}=\alpha I_{s}
\end{gathered}
$$

where $f_{W}$ is Lambert omega function [22], $f_{W}(W)=W e^{W}, I_{\text {sat }}$ is the saturation intensity, and $I_{0}$ is an initial intensity. When the relaxed state $|3\rangle$ is not considered, namely just two channel model, $\alpha=1$. In general, the lifetime from the excited state $|2\rangle$ to the relaxed state $|3\rangle$-which is $\tau_{23}$-is much shorter that the others, so the coefficient $\alpha$ becomes greater than 1 . This leads to the saturation limit $I_{s a t}$ being higher. This situation can be interpreted as due to the quick annihilation of the excited state by the instant relaxation, the number of excited state can be kept small even for high intensity of photons in the pulse. In other words, the relaxed state behaves as a reserver of excited photo electrons. When the intensity $I$ is well above the saturation threshold $I_{\text {sat }}$,

$$
I=I_{0}-\eta,(\eta<1) .
$$


The asymptotic behavior of $I$ is,

$$
I \sim I_{0}+I_{\text {sat }} g_{0} z .
$$

We see that the attenuation of intensity is no longer exponential, but linear.

\section{Appendix B. Short Pulse Limit}

For a short pulse limit, we employ the Frantz-Nodvik model [23]. In this model, it is supposed that the lifetime of an excited state is much longer than the width of pulse, such that it reduces a limit of lifetime to infinity. In the three-channel model, the decay terms are dropped off and the equations have only absorption and stimulated emission processes. Naturally, it reduces to a two-channel model without an intermediate relaxed state. The detail of the derivation can be found in Reference [23]. The final form of the fluence, $\phi(z)=\int I(z, t) d t$, at a position $z$ in one-dimensional model is

$$
\begin{gathered}
\phi(z)=\phi_{\text {sat }} \ln \left[1+e^{g_{0} z}\left(e^{\frac{\phi_{0}}{\phi_{\text {sat }}}}-1\right)\right] \\
\phi_{\text {sat }}=\frac{h v}{2 \sigma} .
\end{gathered}
$$

When $\phi_{0}$ is well above $\phi_{\text {sat }}$, as a long pulse model we get the asymptotic linear behavior,

$$
\phi(z)=\phi_{0}+\phi_{\text {sat }} g_{0} z .
$$

\section{Appendix C. Steady State Equation for TPA}

The equations and solution with TPA case under steady condition in the ground state are:

$$
\begin{gathered}
\frac{d I(z)}{d z}=g_{0} I(z)+\bar{g}_{2} I^{2}(z) \\
I(z)=I_{0} e^{g_{0} z} \frac{1}{1+\frac{\bar{g}_{2}}{g_{0}} I_{0}\left(1-e^{g_{0} z}\right)} \\
\bar{g}_{2}=-\frac{\sigma_{2}}{h v} N
\end{gathered}
$$

\section{References}

1. Ackermann, W.; Asova, G.; Ayvazyan, V.; Azima, A.; Baboi, N.; Bähr, J.; Balandin, V.; Beutner, B.; Brandt, A.; Bolzmann, A.; et al. Operation of a free-electron laser from the extreme ultraviolet to the water window. Nat. Photonics 2007, 1, 336-342.

2. Allaria, E.; Battistoni, A.; Bencivenga, F.; Borghes, R.; Callegari, C.; Capotondi, F.; Castronovo, D.; Cinquegrana, P.; Cocco, D.; Coreno, M.; et al. Tunability experiments at the FERMI@Elettra free-electron laser. New J. Phys. 2012, 14, 113009.

3. Emma, P.; Akre, R.; Arthur, J.; Bionta, R.; Bostedt, C.; Bozek, J.; Brachmann, A.; Bucksbaum, P.; Coffee, R.; Decker, F.J.; et al. First lasing and operation of an Ångstrom-wavelength free-electron laser. Nat. Photonics 2010, 4, 641-647.

4. Ishikawa, T.; Aoyagi, H.; Asaka, T.; Asano, Y.; Azumi, N.; Bizen, T.; Ego, H.; Fukami, K.; Fukui, T.; Furukawa, Y.; et al. A compact X-ray free-electron laser emitting in the sub-ångström region. Nat. Photonics, 2012, 6, 540-544.

5. Bostedt, C.; Boutet, S.; Fritz, D.M.; Huang, Z.; Lee, H.J.; Lemke, H.T.; Robert, A.; Schlotter, W.F.; Turner, J.J.; Williams, G.J. Linac Coherent Light Source: The first five years. Rev. Mod. Phys. 2016, 88, 015007.

6. Ciricosta, O.; Chung, H.K.; Lee, R.W.; Wark, J.S. Simulations of neon irradiated by intense X-ray laser radiation. High Energy Density Phys. 2011, 7, 111-116. 
7. Nagler, B.; Zastrau, U.; Faustlin, R.R.; Vinko, S.M.; Whitcher, T.; Nelson, A.J.; Sobierajski, R.; Krzywinski, J.; Chalupsky, J.; Abreu, E.; et al. Turning solid aluminium transparent by intense soft X-ray photoionization. Nat. Phys. 2009, 5, 693-696.

8. Di Cicco, A.; Hatada, K.; Giangrisostomi, E.; Gunnella, R.; Bencivenga, F.; Principi, E.; Masciovecchio, C.; Filipponi, A. Interplay of electron heating and saturable absorption in ultrafast extreme ultraviolet transmission of condensed matter. Phys. Rev. B 2014, 90, 220303.

9. Rackstraw, D.S.; Ciricosta, O.; Vinko, S.M.; Barbrel, B.; Burian, T.; Chalupský, J.; Cho, B.I.; Chung, H.K.; Dakovski, G.L.; Engelhorn, K.; et al. Saturable absorption of an X-Ray Free-Electron-Laser heated Solid-Density aluminum plasma. Phys. Rev. Lett. 2015, 114, 015003.

10. Yoneda, H.; Inubushi, Y.; Yabashi, M.; Katayama, T.; Ishikawa, T.; Ohashi, H.; Yumoto, H.; Yamauchi, K.; Mimura, H.; Kitamura, H. Saturable absorption of intense hard X-rays in iron. Nat. Commun. 2014, 5, 5080.

11. Kitamura, H. Rate equation for intense core-level photoexcitation and relaxation in metals. J. Phys. B At. Mol. Opt. Phys. 2010, 43, 115601.

12. Kitamura, H. Rapid energy-level shifts in metals under intense inner-shell photoexcitation. High Energy Density Phys. 2012, 8, 66-70.

13. Iglesias, C.A. XUV absorption by solid-density aluminum. High Energy Density Phys. 2010, 6, 311-317.

14. Vinko, S.M.; Gregori, G.; Desjarlais, M.P.; Nagler, B.; Whitcher, T.J.; Lee, R.W.; Audebert, P.; Wark, J.S. Free-free opacity in warm dense aluminum. High Energy Density Phys. 2009, 5, 124-131.

15. Rackstraw, D.S.; Vinko, S.M.; Ciricosta, O.; Chung, H.K.; Lee, R.W.; Wark, J.S. Simulations of the time and space-resolved x-ray transmission of a free-electron-laser-heated aluminium plasma. J. Phys. B At. Mol. Opt. Phys. 2016, 49, 035603.

16. Hatada, K.; Di Cicco, A. Modeling saturable absorption for ultra short X-ray pulses. J. Electron Spectrosc. Relat. Phenom. 2014, 196, 177-180.

17. Courant, R.; Friedrichs, K.; Lewy, H. On the Partial Difference Equations of Mathematical Physics. IBM J. Res. Dev. 1967, 11, 215-234.

18. Almbladh, C.O.; Morales, A.L.; Grossmann, G. Theory of Auger core-valence-valence processes in simple metals. I. Total yields and core-level lifetime widths. Phys. Rev. B, 1989, 39, 3489-3502.

19. Di Cicco, A.; Bencivenga, F.; Battistoni, A.; Cocco, D.; Cucini, R.; D’Amico, F.; Fonzo, S.D.; Filipponi, A.; Gessini, A.; Giangrisostomi, E.; et al. Probing matter under extreme conditions at Fermi@Elettra: The TIMEX beamline. In Damage to VUV, EUV, and X-ray Optics III; Juha, L., Bajt, S., London, R.A., Eds.; SPIE: Prague, Czech Republic, 2011; Volume 8077, p. 807704.

20. Di Cicco, A.; Masciovecchio, C.; Bencivenga, F.; Principi, E.; Giangrisostomi, E.; Battistoni, A.; Cucini, R.; D'Amico, F.; Di Fonzo, S.; Gessini, A.; et al. Probing matter under extreme conditions at the free-electron-laser facilities: the TIMEX beamline. Not. Neutroni E Luce Di Sincrotrone 2013, 18, 19-25.

21. Masciovecchio, C.; Battistoni, A.; Giangrisostomi, E.; Bencivenga, F.; Principi, E.; Mincigrucci, R.; Cucini, R.; Gessini, A.; D'Amico, F.; Borghes, R.; et al. EIS: the scattering beamline at FERMI. J. Synchrotron Radiat. 2015, $22,553-564$.

22. Corless, R.M.; Gonnet, G.H.; Hare, D.E.G.; Jeffrey, D.J.; Knuth, D.E. On the LambertW function. Adv. Comput. Math. 1996, 5, 329-359.

23. Frantz, L.M.; Nodvik, J.S. Theory of Pulse Propagation in a Laser Amplifier. J. Appl. Phys. 1963, 34, 2346-2349, doi:10.1063/1.1702744.

(C) 2017 by the authors. Licensee MDPI, Basel, Switzerland. This article is an open access article distributed under the terms and conditions of the Creative Commons Attribution (CC BY) license (http:// creativecommons.org/licenses/by/4.0/). 